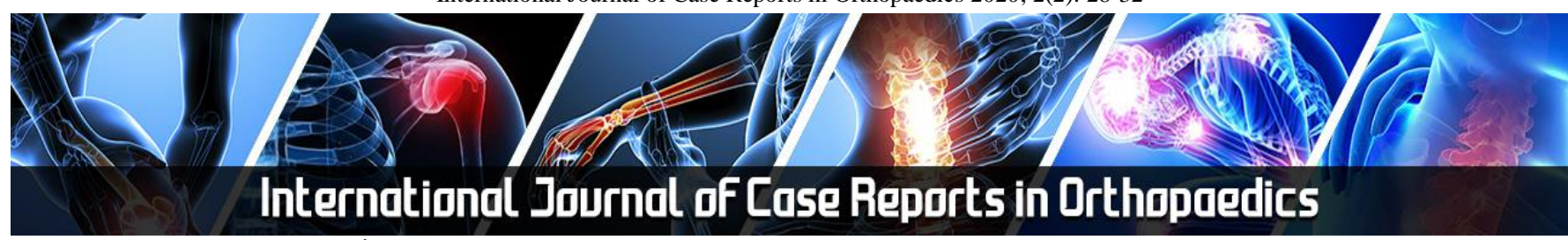

E-ISSN: 2707-8353 P-ISSN: 2707-8345 IJCRO 2020; 2(2): 28-32 Received: 26-05-2020 Accepted: 29-06-2020

Rui Viegas

Hospital Beatriz Ângelo, Loures, Portugal

Pedro Amaro

Hospital Beatriz Ângelo, Loures, Portugal

José Nuno Ferreira Hospital Beatriz Ângelo, Loures, Portugal

José Caldeira

Hospital Beatriz Ângelo, Loures, Portugal

Pedro Falcão Hospital Beatriz Ângelo Loures, Portugal

Carolina Baptista Hospital da Luz, Lisboa, Portugal
Corresponding Author: Rui Viegas

Hospital Beatriz Ângelo Loures, Portugal

\section{Distal radioulnar joint arthroplasty after primary excision of a giant cell tumor of distal ulna: A case report}

\author{
Rui Viegas, Pedro Amaro, José Nuno Ferreira, José Caldeira, Pedro \\ Falcão and Carolina Baptista
}

DOI: https://doi.org/10.22271/27078345.2020.v2.i2a.26

\begin{abstract}
We present a 34-year-old man with a giant cell tumor of the distal ulna. Treatment strategy included en bloc excision and distal radioulnar joint arthroplasty. En bloc excision is a good oncological option in GCT of the distal ulna, preventing local recurrence. Reconstructive procedures after distal ulna excision remain a controversial topic. Distal radioulnar joint arthroplasty can provide an excellent functional result with good range of motion, absence of pain and weight bearing capacity.
\end{abstract}

Keywords: Giant cell tumor; distal ulna; en bloc excision; distal radioulnar arthroplasty

\section{Introduction}

Giant cell tumor (GCT) of bone is a rare and benign neoplasm that represents $3-5 \%$ of all primary bone tumors and $15-20 \%$ of all benign bone tumors ${ }^{[1,3]}$. Although regarded as a benign tumor, it can be locally aggressive and cause substantial bone destruction and mechanical failure ${ }^{[2]}$. Besides, in approximately $2-3 \%$ of cases, metastases can develop, mostly to the lungs. However, these metastases often have relatively indolent behavior ${ }^{[1,3]}$. The distal ulna is an uncommon localization for GCT, which most frequently affects distal femur, proximal tibia and distal radius ${ }^{[2,4]}$.

Surgical resection is the standard of care for the treatment of GCT. Nevertheless, the choice between intralesional curettage and en bloc excision is still controversial ${ }^{[2,5,6]}$. On the one hand, intralesional excision is associated with higher rates of local recurrence. On the other hand, en bloc excision may lead to functional impairment. More specifically, distal ulna resection may be associated with ulnocarpal joint instability, decreased grip strength and stump impingement ${ }^{[7]}$. Distal radioulnar joint (DRUJ) prosthetic reconstruction has been reported to provide good results, minimizing the above-mentioned problems ${ }^{[8,10]}$.

The purpose of this case report is to provide a description of the clinical presentation of a giant cell tumor in a rare location and to present the outcomes at 18-months follow-up of DRUJ arthroplasty as a solution to ensure good wrist function after distal ulna excision.

\section{Case Report}

A 34-year-old male patient employed as a bricklayer presented to the emergency room with a 2-month history of progressive left wrist pain. He had normal wrist range of motion and a tender swelling over the distal end of the ulna.

Radiographs showed a lytic lesion of the distal ulna. An MRI was obtained to further define the extent and nature of the lesion, revealing an expansive mass measuring $30 \mathrm{~mm}$ x $22 \mathrm{~mm}$ $\mathrm{x} 19 \mathrm{~mm}$ that was hypointense in T1-weighted images and heterogeneously hyperintense in T2-weighted images with fat suppression (Figures 1 and 2). These findings were compatible with a giant cell tumor and a surgical biopsy confirmed the suspected diagnosis.

A multidisciplinary team discussed the case and together with the patient decided on wide resection and DRUJ arthroplasty with a multi-component implant (APTIS Medical ${ }^{\circledR}$, Louisville, KY, USA). A preoperative surgical plan was done based on the radiographs and MRI images, allowing customization of the surgery to the specific characteristics of the patient (Figure 3). 


\section{Surgical technique}

The operative procedure was performed with a tourniquet, without exsanguination prior to inflation. The tumor was approached through a $10 \mathrm{~cm}$ long $\mathbf{J}$ shaped incision starting in the dorsal ulna diaphysis and turning radially at the head of the ulna. A retinacular flap based ulnarward and extending radially to the second compartment was elevated to later provide a barrier between the prosthesis and the ECU tendon. The tumor was identified and excised ensuring that there was a cleavage plan with the surrounding structures and that its capsule was intact. The total amount of bone resected was $65 \mathrm{~mm}$ long (Figure 4). To ensure a safe surgical margin, an intraoperative extemporaneous exam was requested. After radial and ulnar preparation according to the surgical technique, the final components were inserted: size 20 radial component and $5 \mathrm{~mm}$ diameter ulnar stem with a $40 \mathrm{~mm}$ extension (Figure 5). An intraoperative assessment of passive range of motion confirmed full pronation and supination of the forearm. The extensor retinaculum was repaired with nonabsorbable sutures and the skin closed with staples. The forearm was immobilized in a sugar tong splint for one week, after which gentle range of motion exercises of the wrist were started. Definitive histological examination reported a giant cell tumor located in the medullary cavity with cortical destruction and adjacent soft tissue involvement. There was absence of vascular invasion, absence of mitotic figures and surgical margins were free from tumor.

\section{Results}

At 18-months follow-up, the patient reports no wrist pain and good range of motion with a slight extension and pronation deficit (Figure 6). Radiographs show good implant alignment, no signs of loosening and no evidence of local tumor recurrence (Figure 7).

\section{Discussion}

GCT most commonly occurs after skeletal maturity, affecting young adults aged 20 to 50 years with a slight female predominance ${ }^{[1,3]}$. Distal ulna is a very rare location for GCT of the bone, accounting for $0.45 \%$ to $3.2 \%$ of all GCT cases ${ }^{[7]}$.

The treatment of GCT encompasses surgical resection and it aims at adequate tumor resection, prevention of local recurrence and preservation of limb function ${ }^{[7,11]}$. Various options have been proposed but there is no consensus regarding the ideal treatment for GCT of the distal ulna ${ }^{[6,7]}$. These options may be divided into two main surgical approaches: intralesional curettage and en bloc excision. The choice of surgical intervention must take into consideration the risk of recurrence and the morbidity of the procedure.

Intralesional curettage preserves joint function but has higher local recurrence rates compared with en bloc excision: $27 \%-65 \%$ after isolated curettage; $0 \%-29 \%$ after curettage with local adjuvant application of polymethylmethacrylate and $0 \%-12 \%$ after en bloc resection ${ }^{[3,6]}$. En bloc resection provides lower recurrence rates at the expense of sacrificing joint function.

Campanacci classified GCT of the bone based on the radiographic findings ${ }^{[2]}$.

- Grade 1 - well-defined margin and an intact cortex;

- Grade 2 - relatively well-defined margin with thinned and moderately expanded cortex;
- Grade 3 - unclear margins, cortical destruction and soft tissue extension.

This classification is useful because it may provide a guide to treatment: intralesional curettage for grade 1 and grade 2 lesions and en bloc resection for grade 3 lesions [1, 7]. However, some authors consider that the classification has a limited role in surgical planning given that it has not been demonstrated that a higher grade correlates with a higher risk of local recurrence ${ }^{[12,13]}$.

The need for reconstructive procedures after distal ulna excision is still controversial. Some authors believe that there is no place for reconstruction after distal ulna excision, reporting good functional outcomes $[11,14,16]$. Other authors believe that resection of distal ulna without reconstruction is associated with ulnocarpal joint instability, unsteady rotation of the carpal unit around the ulnar axis, decreased grip strength and stump impingement $[7,17,19]$. Ultimately, this will lead to pain and poor wrist function that occurs more commonly in young and active patients which constitute the majority of the GCT population.

To minimize the morbidity after distal ulna excision, several reconstructive procedures have been described. Soft tissue procedures include stabilization of the ulnar stump involving flexor carpi ulnaris or extensor carpi ulnaris in an attempt to prevent ulnar subluxation and impingement. These techniques reported pain relief in the majority of the patients but there was no evaluation upon weight loading of the hand ${ }^{[18]}$. Ulnar buttress arthroplasty with iliac crest autograft and ulnar stump stabilization is another reconstructive procedure and has reported good functional outcomes ${ }^{[20]}$.

Following distal ulna excision, DRUJ prosthesis can be an option to reconstruct the joint. There are two main prosthesis types, one that replaces the ulnar head only and a multi-component implant that replaces the ulnar head and the sigmoid notch. According to a systematic review, multicomponent implants appear to have better outcomes in terms of stability, function and longevity but a higher rate of post-operative soft tissue complications comparing to implants that replace the ulnar head only ${ }^{[10]}$. Furthermore, they present good functional outcomes, infrequent complications and an implant survival rate of $97 \%$ at a mean of 56 months ${ }^{[21]}$. In the literature, we found two case reports describing hemi-arthroplasty ${ }^{[22,23]}$ and three case reports describing multi-component arthroplasty after distal ulna excision due to GCT ${ }^{[17,24,25]}$. Although having a maximum follow-up of 2 years, all those cases reported good range of motion wrist function. Apart from an asymptomatic aseptic loosening, no other complications were described.

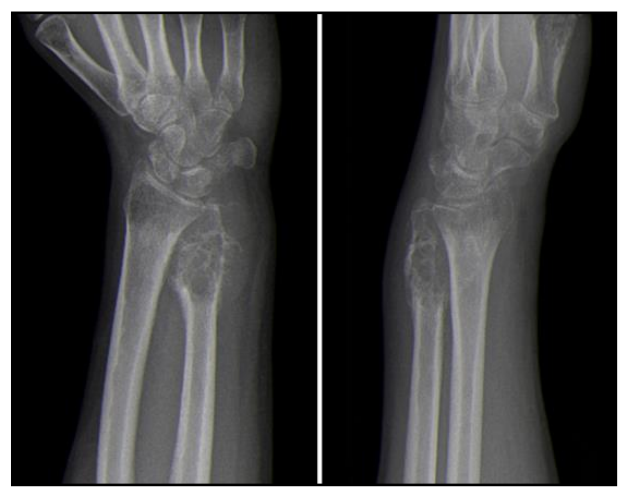

Fig 1: Preoperative radiographs: anteroposterior and lateral views 


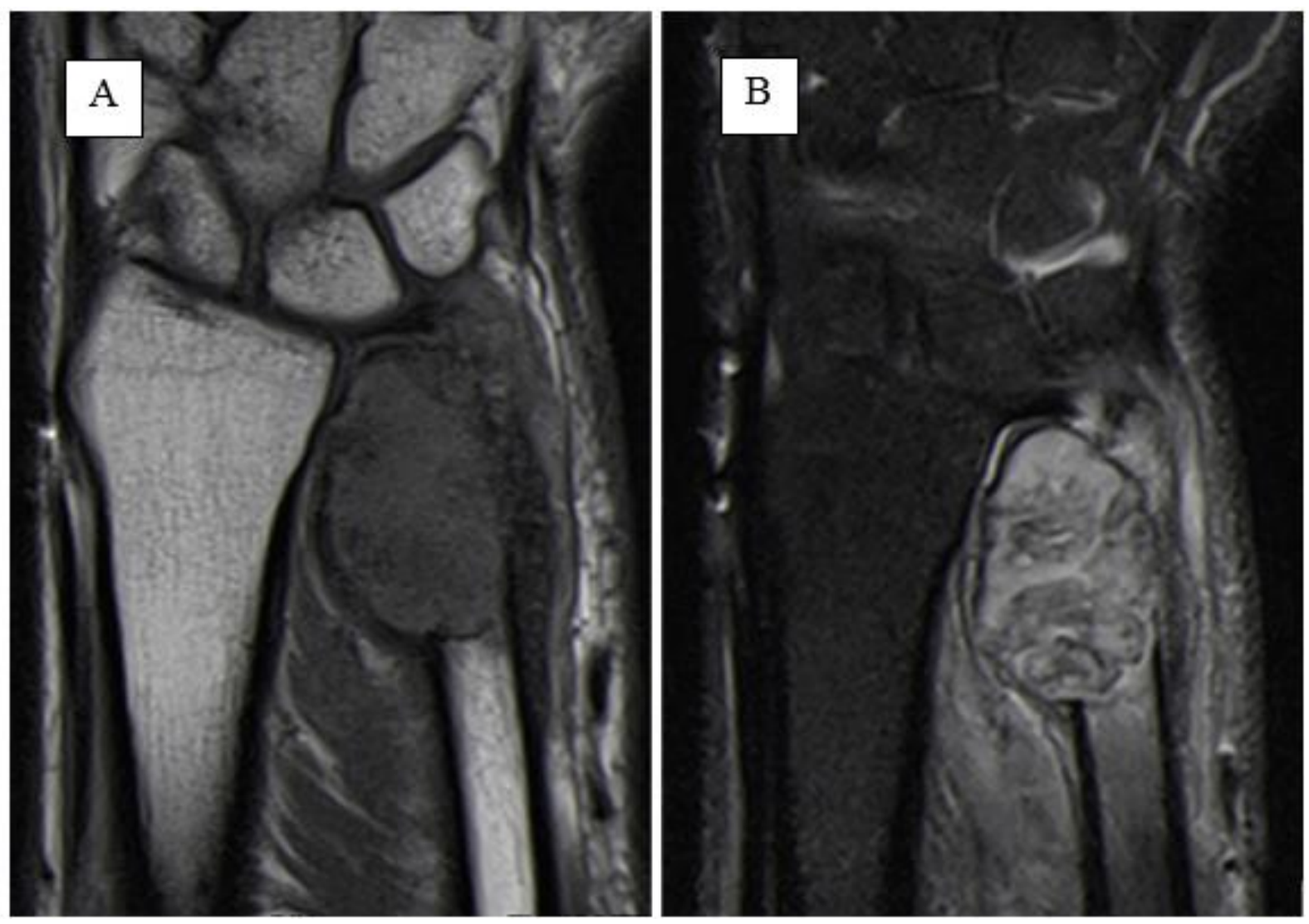

Fig 2: Preoperative MRI revealing a lesion which was hypointense in T1-weighted images (A) and heterogeneously hyperintense in T2weighted images (B) with fat suppression

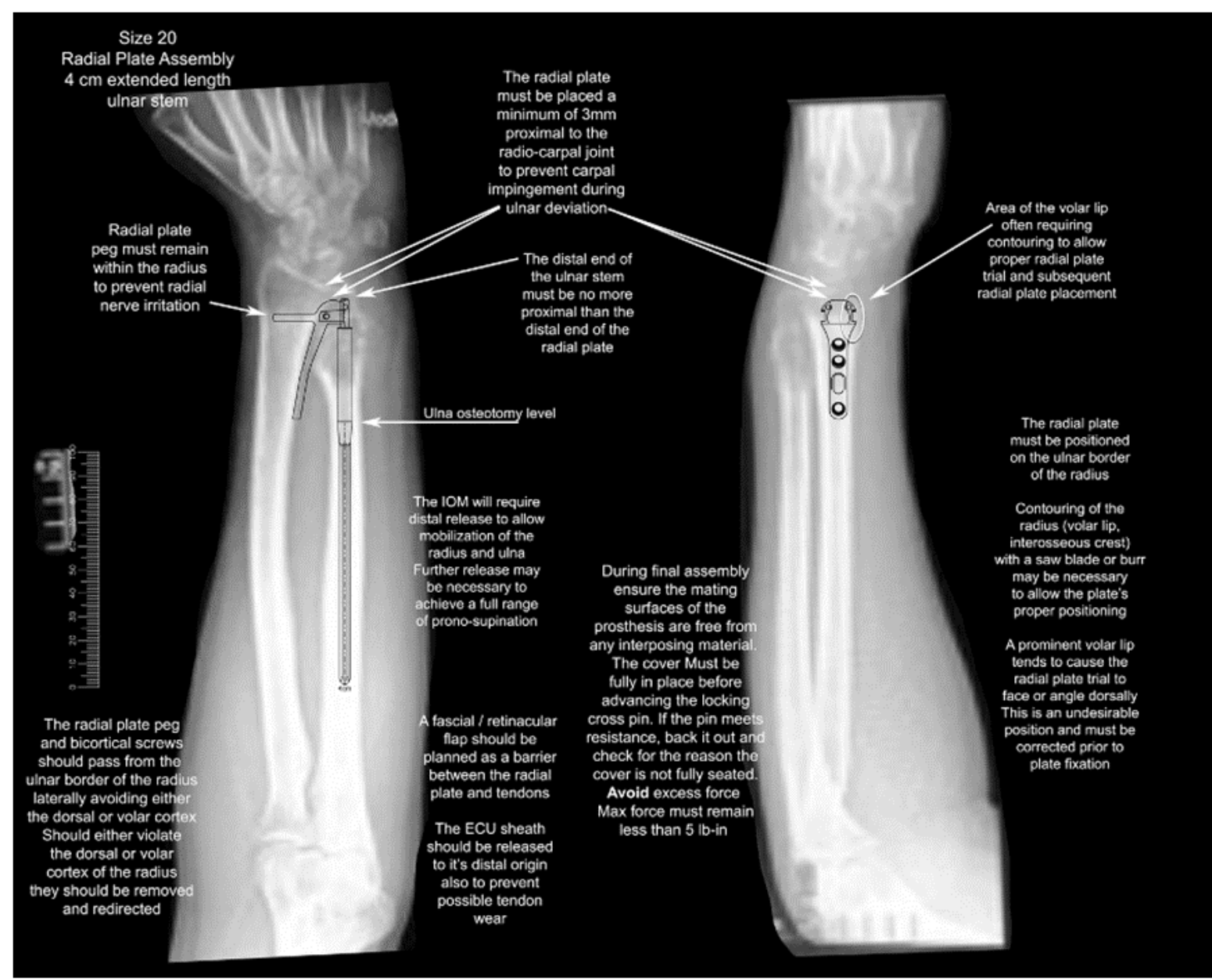

Fig 3: Preoperative template 


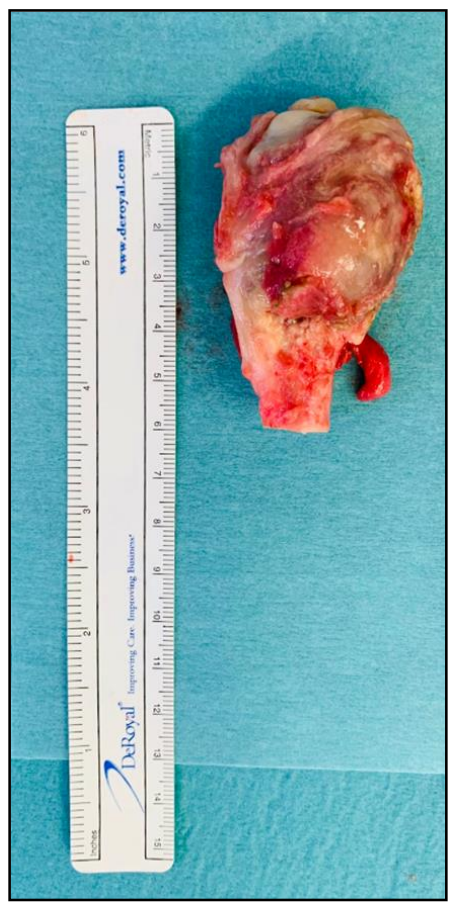

Fig 4: Intraoperative photograph of resected giant cell tumor of the distal ulna

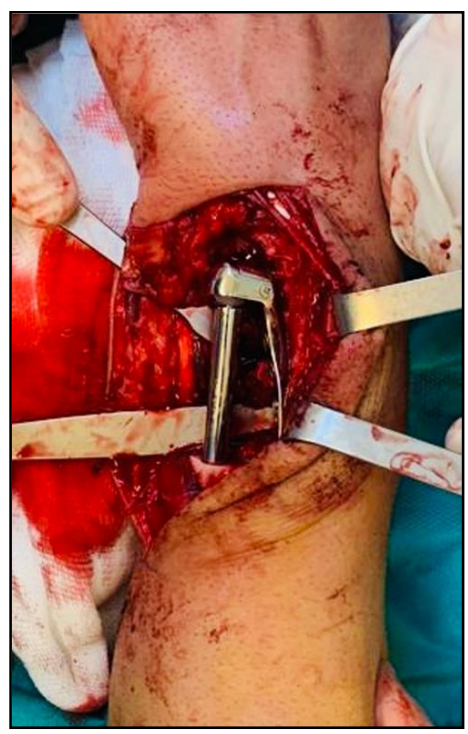

Fig 5: Intraoperative photograph of final implant position (Scheker Distal Radioulnar Joint Prosthesis - APTIS Medical ${ }^{\circledR}$, Louisville, KY, USA)

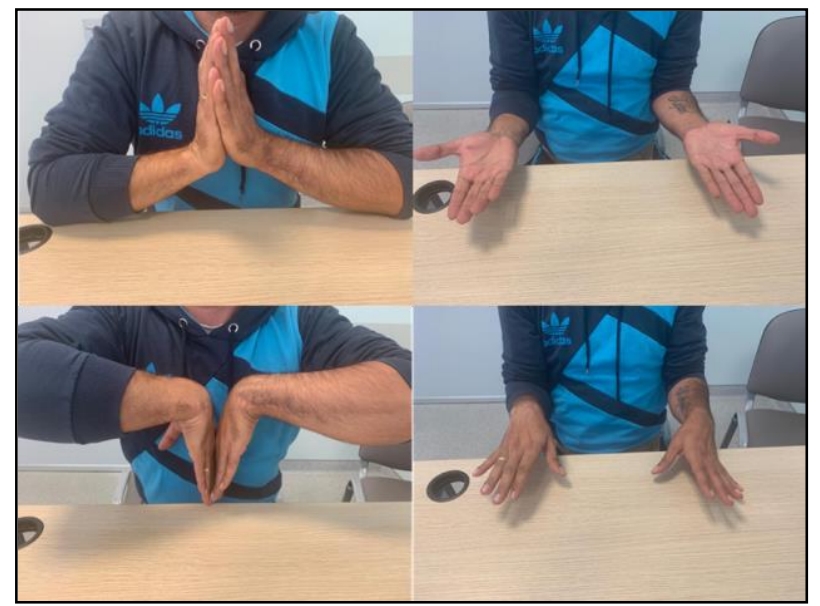

Fig 6: Range of motion at 18-months follow-up

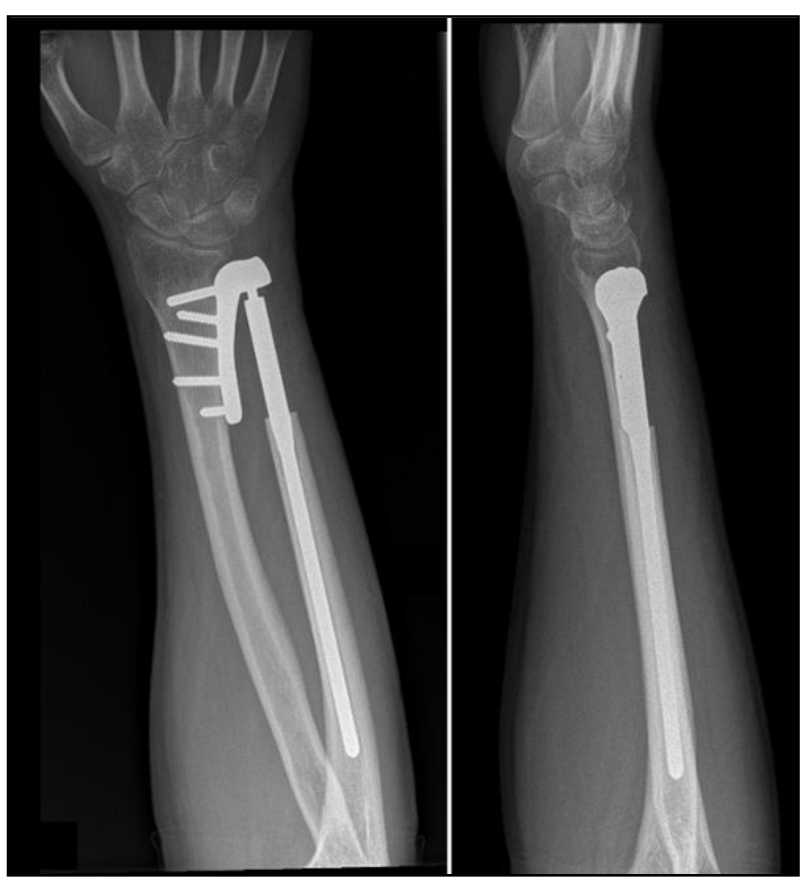

Fig 7: Anteroposterior and lateral radiographs at 18-months follow-up

\section{Conclusion}

This case demonstrates that en bloc excision is a good oncological option in GCT of the distal ulna, preventing local recurrence. Moreover, DRUJ arthroplasty can provide an excellent functional result with good range of motion, absence of pain and weight bearing capacity.

\section{References:}

1. Thomas D, Desai J, Damron T. Giant cell tumor of bone. UpToDate. Accessed 2020, May 5. https://www.uptodate.com/contents/giant-cell-tumor-ofbone.

2. Sobti A, Agrawal P, Agarwala S, Agarwal M. Giant Cell Tumor of Bone - An Overview. The archives of bone and joint surgery. 2016; 4(1):2-9.

3. Heijden L, Dijkstra PDS, Sande MAJ, Kroep JR, Nout RA, Rijswijk CSP, Bovée JVMG et al. The Clinical Approach Toward Giant Cell Tumor of Bone. The Oncologist. 2014; 19(5):550-561.

4. Turcotte RE. Giant Cell Tumor of Bone. Orthopedic Clinics of North America. 2006; 37(1):35-51.

5. Skubitz KM. Giant Cell Tumor of Bone: Current Treatment Options. Curr Treat Options in Oncol. 2014; 15(3):507-518.

6. Zhang J, Li Y, Li D, Xia J, Li S, Yu S et al. Clinical effects of three surgical approaches for a giant cell tumor of the distal radius and ulna. Molecular and Clinical Oncology. 2016; 5(5):613-617.

7. Jamshidi K, Bahrabadi M, Bagherifard A, Mohamadpour M. Surgical treatment outcome of giant cell tumor of distal ulna: En bloc resection vs. curettage and bone graft. Med J Islam Republic Iran. 2018; 32(1):249-253.

8. Moulton LS, Giddins GEB. Distal radio-ulnar implant arthroplasty: a systematic review. J Hand Surg Eur. 2017; 42(8):827-838.

9. Reissner L, Böttger K, Klein H, Calcagni M, Giesen T. Midterm Results of Semiconstrained Distal Radioulnar 
Joint Arthroplasty and Analysis of Complications. Jnl Wrist Surg. 2016; 05(04):290-296.

10. Calcagni M, Giesen T. Distal radioulnar joint arthroplasty with implants: a systematic review. EFORT Open Reviews. 2016; 1(5):191-196.

11. Dhillon MS, Saini R, Gill SS. Is there a need for reconstruction after excision of the distal ulna for Giant-Cell tumour? Acta Orthop Belg. 2010; 76(1):3037.

12. Mavrogenis AF, Igoumenou VG, Megaloikonomos PD, Panagopoulos GN, Papagelopoulos PJ, Soucacos PN et al. Giant cell tumor of bone revisited. SICOT-J. 2017; 3(54).

13. Wang H, Wan N, Hu Y. Giant cell tumour of bone: a new evaluating system is necessary. International Orthopaedics (SICOT). 2012; 36(12):2521-2527.

14. Cooney WP, Damron TA, Sim FH, Linscheid RL. En Bloc Resection of Tumors of the Distal End of the Ulna. The Journal of Bone \& Joint Surgery. 1997; 79(3):406-12.

15. Wolfe SW, Mih AD, Hotchkiss RN, Culp RW, Kiefhaber TR, Nagle DJ et al. Wide excision of the distal ulna: A multicenter case study. The Journal of Hand Surgery. 1998; 23(2):222-228.

16. Dhinsa BS, Gregory JJ, Nawabi DH, Khan S, Pollock $\mathrm{R}$, Aston WJ et al. The outcome of resection of the distal ulna for tumour without soft-tissue or prosthetic reconstruction. The Bone \& Joint Journal. 2014; 96$\mathrm{B}(10): 1392-1395$.

17. Jones NF, Graham DJ. Radical Resection of a Recurrent Giant Cell Tumor of the Distal Ulna and Immediate Reconstruction With a Distal Radio-Ulnar Joint Implant Arthroplasty. HAND. 2020; 0(0):155894471989577.

18. Laurentin-Pérez LA, Goodwin AN, Babb BA, Scheker LR. A Study of Functional Outcomes Following Implantation of A Total Distal Radioulnar Joint Prosthesis. J Hand Surg Eur. 2008; 33(1):18-28.

19. Shaaban H, Giakas G, Bolton M, Williams R, Scheker LR, Lees VC et al. The distal radioulnar joint as a loadbearing mechanism-a biomechanical study. The Journal of Hand Surgery. 2004; 29(1):85-95.

20. Tripathy S, Naik M, Sujir P, Rao S. Ulnar buttress arthroplasty after en bloc resection of a giant cell tumor of the distal ulna. Indian J Orthop. 2013; 47(2):211-14.

21. Moulton LS, Giddins GEB. Distal radio-ulnar implant arthroplasty: a systematic review. J Hand Surg Eur Vol. 2017; 42(8):827-838.

22. Burke CS, Gupta A, Buecker P. Distal Ulna Giant Cell Tumor Resection with Reconstruction Using Distal Ulna Prosthesis and Brachioradialis Wrap Soft Tissue Stabilization. HAND. 2009; 4(4):410-414.

23. Roidis NT, Gougoulias NE, Liakou PD, Malizos KN. Distal Ulnar Implant Arthroplasty as a Definitive Treatment of a Recurrent Giant-Cell Tumor. The Journal of Hand Surgery. 2007; 32(8):1262-1266.

24. Gracia I, Proubasta IR, Trullols L, Peiró A, Moya E, Cortés $\mathrm{S}$ et al. Distal radioulnar joint prosthesis for the treatment of giant cell tumor of the distal ulna: a case report and literature review. Strategies in Trauma and Limb Reconstruction. 2011; 6(2):103-106.

25. Pirela-Cruz MA, Higgs M, Reddy K, Abdelfattah H, Cameron C, Hakim MN CR et al. Treatment of a Giant
Cell Tumor of the Distal Ulna with a Fully Constrained Prosthesis. El Paso Phys Mag. 2008; 31(3):9-13. 